\title{
Morus alba L. extracts inhibit adipocyte differentiation but stimulate lipolysis in 3T3-L1cells
}

\author{
G. H. Kim and H. S. Park \\ Plant Resources Reserach Institute, Duksung Women's University, Korea
}

Fruits of mulberry (Morus alba L.) belonging to the Moraceae family have been widely used for therapeutic purposes in Asian countries for centuries. Adipocyte differentiation has often been a target of anti-obesity strategies because obesity is caused not only by hypertrophy of adipocytes, but also by adipocyte hyperplasia ${ }^{(1)}$. The 3T3-L1 cell line is commonly used as an adipocyte differentiation model system for investigation of molecular mechanisms that regulate adipogenesis ${ }^{(2)}$. This study was performed to investigate the effects of ethanol extract and fractions of $M$. alba L. on adipocyte differentiation in 3T3-L1cells. The anti-adipogenic effect of $M$. alba L. extracts was examined for its effect on the anti-adipocyte differentiation of 3T3-L1 cells by Oil red O staining assay. The lipolysis effect of $M$. alba L. was measured by glycerol releases assay.

A

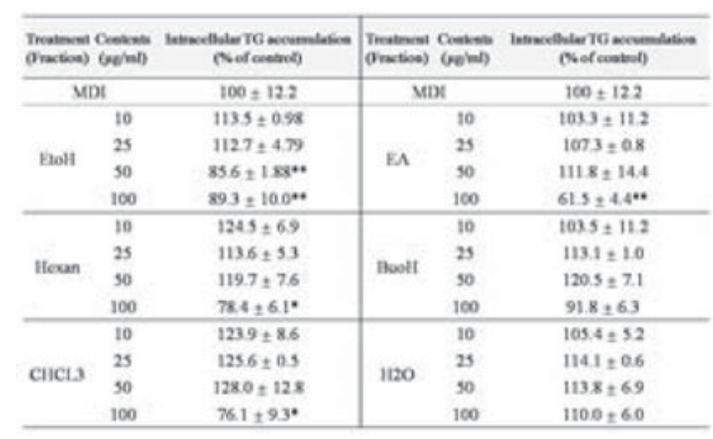

B

\begin{tabular}{|c|c|c|c|c|c|}
\hline \multicolumn{3}{|c|}{ 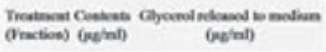 } & $\begin{array}{l}\text { Theations } \\
\text { (ination) }\end{array}$ & \multicolumn{2}{|c|}{ 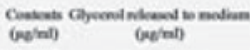 } \\
\hline $\mathrm{Me}$ & & $6.56 \div 0.23$ & $\mathrm{MD}$ & & $5.61=0.14$ \\
\hline \multirow{4}{*}{ Enoll } & 10 & $6.50 \div 0.43$ & \multirow{4}{*}{ EA } & 10 & $5.66 \pm 0.13$ \\
\hline & 25 & $6.75 \div 0.44$ & & 25 & $5.49 \pm 0.12$ \\
\hline & 50 & $6.82 \div 0.17$ & & 50 & $533=0.05 \%$ \\
\hline & 100 & $636: 077$ & & 100 & $553 \div a .22$ \\
\hline \multirow{4}{*}{ Heun } & 10 & $3.46 \div 0.91$ & \multirow{4}{*}{ Buett } & 10 & $5.58 \div 0.10$ \\
\hline & 25 & $455=0.20^{\circ}$ & & 25 & $5.43 \div 0.07$ \\
\hline & 50 & $4.97 \pm 0.53 *$ & & 90 & $5.10 \pm 092$ \\
\hline & 100 & $3.63+0.36 * t$ & & 100 & $5.15 \div 0.45$ \\
\hline \multirow{4}{*}{ chess } & 10 & $5.22 \div 0.93$ & \multirow{4}{*}{120} & 10 & $530 \div 0.15$ \\
\hline & 25 & $3.91 \pm 0.43 * *$ & & 25 & $3.34 \div 0.31$ \\
\hline & so & $4.19+1.11 *$ & & 90 & $360 \div 0.46$ \\
\hline & 100 & $4.41+0.20^{\circ *}$ & & 100 & $5.60 \div 0.25$ \\
\hline
\end{tabular}

Values are means for three independent experiments. Mean values were significantly different from those for MDI group (ANOVA, followed by Duncan test): $* P<0.05, \dagger P<0.01$.

Treatment of 3T3-L1 cells with ethanolic extracts of $M$. alba decreased adipocyte differentiation in a dose-dependent manner (A). Most of the tested fractions exhibited anti-adipogenic effects except for butanol and water-soluble fractions. The ethylacetate-soluble fraction was found to have the greatest inhibitory effect, as indicated by suppression of the lipid accumulation by $38.5 \%$ in response to treatment with $100 \mu \mathrm{g} / \mathrm{ml}$.

On other hands, glycerol secretion decreased in treated-concentration of hexan and chloroform-soluble fraction. The low secretion level of glycerol is not an expected result because it was thought that adipocyte reducing effects are caused by lipolysis. The results indicate that $M$. alba L. is a possible candidate for regulating lipid accumulation in obesity.

1. Caro JF, Dohm LG, Pories WJ et al. (1989) Diab Metab Rev 5, 665-689.

2. Jae Eun K \& Jie C (2004) Diabetes 53, 2748-2756. 